\title{
Stroke prevention by direct revascularization for patients with adult-onset moyamoya disease presenting with ischemia
}

\author{
Tackeun Kim, MD, ${ }^{1}$ Chang Wan Oh, MD, PhD, ${ }^{1}$ O-Ki Kwon, MD, PhD, ${ }^{1}$ Gyojun Hwang, MD, PhD, ${ }^{1}$ \\ Jeong Eun Kim, MD, PhD, ${ }^{2}$ Hyun-Seung Kang, MD, PhD, ${ }^{2}$ Won-Sang Cho, MD, ${ }^{2}$ and \\ Jae Seung Bang, MD'
}

${ }^{1}$ Department of Neurosurgery, Seoul National University Bundang Hospital, Seongnam, Gyeonggi; and 2Department of
Neurosurgery, Seoul National University Hospital, Seoul, Republic of Korea

OBJECTIVE Moyamoya disease (MMD) is a progressive disease that can cause recurrent stroke. The authors undertook this retrospective case-control study with a large sample size in an attempt to assess the efficacy of direct or combined revascularization surgery for ischemia in adults with MMD.

METHODS The authors investigated cases involving patients with moyamoya disease presenting with ischemia who visited Seoul National University Bundang Hospital and Seoul National University Hospital between 2000 and 2014. Among 441 eligible patients, 301 underwent revascularization surgery and 140 were treated conservatively. Variables evaluated included age at diagnosis, sex, surgical record, Suzuki stage, and occurrence of stroke. Patients were stratified into 2 groups based on whether or not they had undergone revascularization surgery. Actuarial 1-, 5-, and 10-year stroke rates were calculated using the life table method. Risk factor analysis for 5 -year stroke occurrence was conducted with multivariate regression.

RESULTS Of the 441 patients, 301 had been surgically treated (revascularization group) and 140 had not (control group). The mean follow-up durations were 45 and 77 months, respectively. The actuarial 10 -year cumulative incidence rate for any kind of stroke was significantly lower in the revascularization group (9.4\%) than in the control group (19.6\%) $(p=0.041)$; the relative risk reduction (RRR) was also superior $(52.0 \%)$ in the revascularization group, and the number needed to treat was 10 . The 10 -year rate of ischemic stroke was greater $(13.3 \%)$ in the control group than in the revascularization group (3.9\%) ( $p=0.019)$. The RRR for ischemic stroke in the revascularization group was $70.7 \%$, and the number needed to treat was 11 . However, the actuarial 1 - and 5 -year rates of ischemic stroke did not significantly differently between the groups. Overall, revascularization surgery was shown to be an independent protective factor, as revealed by multivariate analysis.

CONCLUSIONS Direct or combined revascularization for patients with adult-onset moyamoya disease presenting with ischemia can prevent further stroke.

http://thejns.org/doi/abs/10.3171/2015.6.JNS151105

KEY WORDS bypass surgery; cerebrovascular disease; moyamoya disease; prevention; stroke; vascular disorders

$\mathrm{M}$ oyamoya disease (MMD) is a progressive disease that causes recurrent stroke through progressive stenosis of the terminal internal carotid arteries..$^{19}$ However, the pathogenesis of MMD has not yet been well characterized. Nevertheless, revascularization surgery for symptomatic MMD is considered standard treatment for preventing further stroke, as it results in an augmentation of cerebral blood flow and stabilizes collateral vessels. ${ }^{9,24}$
Due to the progressive nature of MMD, investigations into its natural history have been limited in asymptomatic patients with a relatively stable hemodynamic status. While the annual stroke rate of nonsurgically treated adult patients with MMD has been reported to be 3.2\%$13.3 \%, 3,4,11,13,15,18,23$ the incidence of stroke after direct or combined revascularization surgery appears to be lower. Several retrospective cohort studies have investigated this

ABBREVIATIONS ARR = absolute risk reduction; AS = any kind of stroke; DSA = digital subtraction angiography; D-SPECT = SPECT with acetazolamide (Diamox) challenge; $\mathrm{HS}=$ hemorrhagic stroke; IS = ischemic stroke; $\mathrm{MMD}=$ moyamoya disease; $\mathrm{mRS}=$ modified Rankin Scale; NNT = number needed to treat; $\mathrm{OA}=$ occipital artery; RRR = relative risk reduction; SPECT = single photon emission tomography; STA = superficial temporal artery; TIA = transient ischemic attack.

SUBMITTED May 12, 2015. ACCEPTED June 30, 2015.

INCLUDE WHEN CITING Published online December 4, 2015; DOI: 10.3171/2015.6.JNS151105. 
phenomenon. Among patients with adult-onset MMD, the annual stroke rate after surgery has been reported to be $0.41 \%-4.5 \%(0.14 \%-4.5 \%$ for ischemic stroke [IS], 0\%$1.23 \%$ for hemorrhagic stroke $[\mathrm{HS}])^{2,8,10,20}$ Because these reports are rather variable, it is hard to determine the true efficacy of direct revascularization for adult MMD. Due to the rare and progressive nature of the disease, designing a randomized controlled study with patients with MMD poses even more of a challenge.

In an attempt to overcome these issues, we designed a retrospective case-control study using data from a large number of patients. The aim was to assess the efficacy of direct or combined revascularization surgery for adult MMD by comparing stroke incidence in patients who underwent surgery with the incidence in patients who were treated nonsurgically.

\section{Methods \\ Patients}

We searched our electronic medical record archive to identify eligible patients who were seen at Seoul National University Bundang Hospital or Seoul National University Hospital between December 2000 and March 2014. All patients who met the following criteria were included in the revascularization group: 1) diagnosis of definite or probable MMD via digital subtraction angiography $(\mathrm{DSA})^{9}$ at the age of 18 years or older; 2) presentation with symptoms related to ischemia, such as transient ischemic attack (TIA) or cerebral infarction; 3) having undergone direct or combined revascularization surgery for the treatment of MMD; and 4) having available clinical followup information for more than 6 months postsurgery. We identified a total of 301 surgery patients who met these criteria and were treated between December 2000 and March 2014. In patients who underwent bilateral surgery, duration of follow-up was counted from the time of the first operation.

We investigated age at the time of diagnosis, sex, and surgical records. The initial Suzuki stage was assessed using DSA. The patients' clinical status was evaluated using the modified Rankin Scale (mRS); scores from the time of diagnosis, the 6-month follow-up visit, and the most recent follow-up visit were used in this analysis. The patency of the bypass pedicle was assessed using DSA, CT, and MRI. Discrete information about recurrent stroke was obtained from medical records and radiological examinations.

For the control group, we enrolled patients who met criteria 1) and 2), as described above, but had not undergone revascularization surgery. In our institute, revascularization surgery was considered indicated for patients presenting with symptoms related to hemodynamic impairment, as revealed by single photon emission tomography with acetazolamide (Diamox) challenge (D-SPECT). Routine follow-up was recommended if patients' basal perfusion status was normal or mildly decreased and their vascular reserve capacity in D-SPECT did not decrease by more than $50 \%$ of basal perfusion. Data obtained for the controls included age at the time of diagnosis, sex, initial presentation, Suzuki stage, and development of stroke. In total, 140 patients with available clinical data were included in the control group. These patients were diagnosed and followed in the same 2 institutions as the patients in the revascularization group.

\section{Definition of End Points}

In this study, any kind of stroke (AS) was defined as a symptomatic stroke that was newly developed after revascularization or initiation of observation. The first event of AS was defined as the primary endpoint. AS was additionally divided into IS and HS according to follow-up CT or MRI results. A cerebrovascular event was defined as ischemic symptoms that developed after surgery, irrespective of abnormal imaging findings such as TIA or hyperperfusion syndrome. The perioperative period was defined as 30 days after revascularization.

\section{Surgical Procedures}

All surgically treated patients underwent direct_revascularization-with or without the addition of an indirect revascularization procedure-after confirmation of hemodynamic insufficiency by D-SPECT. For direct anastomosis, the superficial temporal artery (STA) was used as the donor artery in the majority of cases $(n=300,99.7 \%)$. The occipital artery (OA) was used as the donor artery for the remaining $0.3 \%$ of cases $(n=1)$. The cortical branches of the middle cerebral artery $\left(\mathrm{M}_{4}\right)$ were selected as the recipients in all cases.

During the study period, 5 neurosurgeons performed various methods of combined indirect revascularization. The indirect method was selected based on the distribution of the STA and surgeon preferences. Encephalogaleodurosynangiosis was most commonly performed $(\mathrm{n}=$ $148,49.2 \%$ ), followed by encephaloduroarteriosynangiosis $(\mathrm{n}=106,35.2 \%)$ and encephalogaleosynangiosis $(\mathrm{n}=$ $19,6.3 \%)$. Nine $(3.0 \%)$ and $8(2.7 \%)$ of the patients were treated with encephalomyosynangiosis and encephaloduroarteriomyosynangiosis, respectively. Five (1.7\%) of the patients each underwent double-barrel bypass and direct bypass only. One patient $(0.3 \%)$ was treated with an OA to $\mathrm{M}_{4}$ bypass with encephaloduroarteriosynangiosis.

\section{Data Analysis}

Statistical analysis was conducted using $\mathrm{R}$ (Version 3.1.1, 2014; The R Foundation for Statistical Computing, http://www.r-project.org). Continuous variables were expressed as mean \pm standard deviation.

The annual stroke rate was calculated using the following equation:

$$
\begin{aligned}
& \text { Annual Stroke Rate }(\%)= \\
& \qquad\left[(1+\text { Total Stroke Rate })^{\left(\frac{12}{\text { Mean follow-up period (months })}\right)}-1\right] * 100
\end{aligned}
$$

To evaluate stroke risk during specific periods, actuarial 1-year, 5-year, and 10-year stroke rates were calculated using the life table method, and are presented as mean \pm standard error. These rates were compared between groups after standardization to a $\mathrm{Z}$ distribution using the equation shown below. Psurgery and SEsurgery represent 
the stroke rate and standard error of the revascularization group, respectively. Pcontrol and SEcontrol represent the stroke rate and standard error of the control group.

$$
Z=\frac{(1-\text { Psurgery })-(1-\text { Pcontrol })}{\sqrt{\text { SEsurgery }^{2}+\text { SEcontrol }^{2}}}
$$

Finally, using the Z-distribution curve, $1-p(Z)$ was calculated as a $\mathrm{p}$ value to test the hypothesis that the stroke recurrence rate after revascularization of MMD (revascularization group) is lower than that in hemodynamically stable MMD (control group). If this difference in stroke rate was statistically significant, absolute risk reduction (ARR), relative risk reduction (RRR), and the number needed to treat (NNT) were calculated using the following equations: $\mathrm{ARR}=$ (stroke risk of the control group) (stroke risk of the revascularization group); RRR = ARR/ (stroke risk of the control group); and NTT $=1 / \mathrm{ARR}$.

Risk factor analysis for 5-year AS was conducted for the patients with 5 years or more of follow-up data (201 patients; 119 in the revascularization group and 82 in the control group). After univariate analysis using binominal logistic regression, the variables were substituted into multivariate analysis.

\section{Results}

\section{Revascularization Group}

The mean age of patients in the revascularization group at the time of diagnosis was $38.3 \pm 11.6$ years, and $62.1 \%$ were female. Of the 301 patients, $184(61.1 \%)$ presented with TIA without infarction and 115 (38.2\%) presented with IS (Table 1). The initial clinical status was mRS 1 for most patients $(\mathrm{n}=152,50.5 \%)$ followed by $\mathrm{mRS} 0(\mathrm{n}=99$, $32.9 \%)$ and $\mathrm{mRS} 2(\mathrm{n}=41,13.6 \%)$.

Perioperative cerebrovascular events occurred in 149 patients (49.5\%). Among these patients, 8 (2.7\%) developed AS, including $4(1.3 \%)$ IS and $4(1.3 \%)$ HS cases. One of the 4 patients who experienced HS died due to massive postoperative epidural hemorrhage. The crude mortality rate was calculated to be $0.3 \%$.

Perioperative wound problems, including discoloration, dehiscence, or superficial infection, occurred in 20 (6.6\%) cases. Among these, 8 (2.7\%) patients underwent additional surgical procedures while others improved via meticulous wound care. Four (1.3\%) patients experienced postoperative seizures, which were well controlled with antiepileptic drugs.

Clinical status at the final visit was better than the initial status. Most patients $(\mathrm{n}=203,67.4 \%)$ were found to have an $\mathrm{mRS}$ score of 0 at the final visit. Fifty-three $(17.6 \%)$ and $28(9.3 \%)$ had $\mathrm{mRS}$ scores of 1 and 2, respectively. At a mean follow-up of 45 months, the bypass patency rate was $94.7 \%$.

The patency of the bypass pedicle was checked at 6 and 12 months after surgery. The patency rate at 6 months postsurgery was $97.8 \%(\mathrm{n}=276)$. The majority of assessments were based on DSA (77.5\%), followed by MR angiography (20.7\%) and CT angiography (1.8\%). The 1-year
TABLE 1. Summary of characteristics for each group

\begin{tabular}{lcc}
\hline & $\begin{array}{c}\text { Revascularization } \\
\text { Group }\end{array}$ & $\begin{array}{c}\text { Control } \\
\text { Group }\end{array}$ \\
\hline No. of patients & 301 & 140 \\
\hline Mean age at diagnosis (yrs) & $38.3 \pm 11.6$ & $41.1 \pm 11.8$ \\
\hline$<40$ & 169 & 63 \\
\hline$\geq 40$ & 132 & 77 \\
\hline Suzuki stage & & \\
\hline 2 & 29 & 0 \\
\hline 3 & 128 & 12 \\
\hline 4 & 110 & 98 \\
\hline 5 & 26 & 24 \\
\hline 6 & 8 & 0 \\
\hline Sex & 114 & 48 \\
\hline Male & 187 & 92 \\
\hline Female & $44.9 \pm 34.4$ & $77.0 \pm 67.1$ \\
\hline Mean duration of follow-up (mos) & &
\end{tabular}

patency rate was measured as $94.2 \%(\mathrm{n}=104)$, which was verified by MR angiography (in $81.7 \%$ of cases), DSA (in 13.5\%), and CT angiography (in 4.8\%). Because none of the 10 patients with pedicle occlusion experienced a stroke, development of AS did not seem to be related to bypass patency ( $\mathrm{p}=0.570$, Fisher exact test)

\section{Control Group}

A total of 140 patients (mean age at diagnosis $41.1 \pm$ 11.8 years) were included in the control group. As in the revascularization group, female predominance was observed (69.2\%). The mean duration of follow-up in the control group was 77 months. Among the 140 patients, 21 $(15.0 \%)$ had undergone revascularization for the treatment of aggravated clinical symptoms related to hemodynamic compromise at a mean time from diagnosis of 49 months. In these cases, the follow-up period was recorded as censored at the time of surgery.

\section{Comparison of End Points Between Groups}

AS had occurred in $36(8.2 \%)$ of the 441 patients during the follow-up period; 20 patients $(4.5 \%)$ presented with IS and $16(3.6 \%)$ presented with HS. Overall, the 1-, 5-, and 10-year actuarial AS rates were 4.5\%, 6.9\%, and $14.6 \%$, respectively (IS, $2.6 \%, 4.0 \%$, and $8.7 \%$; HS, $1.9 \%$, $2.9 \%$, and $6.0 \%$ ). Within the revascularization group, AS had developed in $18(6.0 \%)$ patients, including 9 IS (3.0\%) and 9 HS (3.0\%) cases, at a mean follow-up of 45 months. By contrast, AS had occurred in 18 patients $(12.9 \%)$ at a mean follow-up of 77 months in the control group. Eleven (7.9\%) of the control patients had experienced IS and 7 (5.0\%) had experienced HS. The annual rates of AS were calculated to be $1.6 \%$ in the revascularization group and $1.9 \%$ in the control group. After revascularization, the annual rates of both IS and HS were $0.8 \%$, whereas the corresponding rates were $1.2 \%$ and $0.8 \%$ in the control group.

The 1- and 5-year actuarial IS and HS rates were not significantly lower in the revascularization group than in the control group. However, the 10-year actuarial AS rate 
was lower in the revascularization group (9.4\%) than in the control group $(19.6 \%, \mathrm{p}=0.041)$. The calculated ARR was $10.2 \%$ and the RRR was $52.0 \%$. The NNT was 10 for 10 -year AS. Similar results were found for IS (3.9\% for the revascularization group vs $13.3 \%$ for the control group, $\mathrm{p}$ $=0.019$ ). The RRR for IS was 70.7\%. Considering ARR (9.4\%), NNT was estimated as 11. However, the risk of HS was similar in the 2 groups across 10 years $(5.6 \%$ for the revascularization group vs $6.5 \%$ for the control group, $\mathrm{p}=$ $0.413)$. These results are shown in Table 2.

A total of 201 patients were followed up for longer than 5 years. The crude rate of AS at the 5-year follow-up was $5.0 \%(2.5 \%$ for the revascularization group and $8.5 \%$ for the control group). For these 201 cases, multivariate risk factor analysis for the 5-year AS rate was performed to adjust for confounders (Table 3). Regression results revealed revascularization surgery to be an independent protective factor regardless of age, sex, or Suzuki stage (OR 0.160, p $=0.031$ ).

\section{Discussion}

While the precise etiology of MMD is yet to be elucidated, it seems clear that it is a progressive disease that can cause further TIA, infarction, or hemorrhage. ${ }^{18}$ Nevertheless, no effective medical treatment modalities for the intracranial arteriopathy of MMD have been established. Although the use of antiplatelet agents has been recommended for patients with MMD, scientific support for this strategy has been lacking. ${ }^{16,26}$ In a recent article, Cho et al. reviewed publications that have reported stroke recurrence in patients who received nonsurgical treatment for MMD. ${ }^{4}$ The authors found that the weighted average of annual stroke rates for a total of 470 patients receiving conservative treatment was 7.1\%. However, to our knowledge, nonsurgical treatments have not yet been compared with surgical treatments on such a large scale.

Surgical flow augmentation has been generally accepted as the most efficient treatment modality for preventing further stroke and disabilities in MMD patients. ${ }^{2,5,8,10,12,17,22}$ Because of the low prevalence of MMD, most studies concerned with surgical outcomes have been conducted with relatively small sample sizes. As far as we know, the largest cohort study, involving 802 patients, was conducted in China. ${ }^{6}$ However, most revascularization reported in this paper was accomplished by means of an indirect method, such as encephaloduroarteriosynangiosis. Examining the results from direct bypass surgery, Guzman et al. reported a $5.5 \%$ risk of AS at the 5-year follow-up, which is very similar to our own results $(5.6 \%){ }^{12}$ Because the majority of patients had undergone direct revascularization, their report may support our findings. On the other hand, several studies have made indirect comparisons of stroke recurrences between surgically and nonsurgically treated patients. For instance, Gross et al. reported the stroke rate in the pretreatment period to be $11 \%$ per hemisphere-year (8.9\% for IS and $2.0 \%$ for HS). After direct bypass surgery, these rates were reduced to $4.5 \%$ (4.5\% for IS and $0 \%$ for HS). ${ }^{10}$ The annual stroke rate seemed to be higher than the ones we report, which could be due to the relatively short-term follow-up in their study (mean 1.3 years). Because this comparison was not made among independent patient groups, the results are not able to show the efficacy of direct revascularization compared with nonsurgical treatment.

In this investigation, we confirmed that a long-term benefit of revascularization exists for patients with adultonset MMD presenting with ischemia. However, the rate

TABLE 2. Stroke recurrence rates*

\begin{tabular}{|c|c|c|c|c|c|c|}
\hline \multirow[b]{2}{*}{ Description } & \multicolumn{2}{|c|}{ Group } & \multirow[b]{2}{*}{$p$ Value } & \multirow[b]{2}{*}{ ARR } & \multirow[b]{2}{*}{ RRR } & \multirow[b]{2}{*}{ NNT } \\
\hline & Revascularization & Control & & & & \\
\hline \multicolumn{7}{|c|}{ Annual stroke rate ( $\%$ per yr) } \\
\hline All stroke & 1.6 & 1.9 & & & & \\
\hline Ischemic stroke & 0.8 & 1.2 & & & & \\
\hline Hemorrhagic stroke & 0.8 & 0.8 & & & & \\
\hline \multicolumn{7}{|l|}{ 1-yr stroke rate $(\%)$} \\
\hline All stroke & $5.1 \pm 1.3$ & $2.9 \pm 1.4$ & 0.875 & - & - & - \\
\hline Ischemic stroke & $2.7 \pm 2.0$ & $2.2 \pm 1.2$ & 0.626 & - & - & - \\
\hline Hemorrhagic stroke & $2.4 \pm 0.9$ & $0.7 \pm 0.7$ & 0.932 & - & - & - \\
\hline \multicolumn{7}{|l|}{5 -yr stroke rate $(\%)$} \\
\hline All stroke & $5.6 \pm 1.4$ & $8.5 \pm 2.9$ & 0.184 & - & - & - \\
\hline Ischemic stroke & $2.7 \pm 1.0$ & $5.9 \pm 2.5$ & 0.117 & - & - & - \\
\hline Hemorrhagic stroke & $2.7 \pm 1.0$ & $2.6 \pm 1.5$ & 0.566 & - & - & - \\
\hline \multicolumn{7}{|l|}{$10-y r$ stroke rate $(\%)$} \\
\hline All stroke & $9.4 \pm 3.1$ & $19.6 \pm 5.0$ & $0.041 \dagger$ & $10.2 \%$ & $52.0 \%$ & 10 \\
\hline Ischemic stroke & $3.9 \pm 1.5$ & $13.3 \pm 4.3$ & $0.019 \dagger$ & $9.4 \%$ & $70.7 \%$ & 11 \\
\hline Hemorrhagic stroke & $5.6 \pm 2.8$ & $6.2 \pm 3.7$ & 0.413 & - & - & - \\
\hline
\end{tabular}


TABLE 3. Risk factor analysis for 201 patients who had been followed up for more than 5 years*

\begin{tabular}{lccllccc}
\hline \multirow{2}{*}{ Variable } & \multicolumn{2}{c}{ Univariate } & & \multicolumn{3}{c}{ Multivariate } \\
\cline { 2 - 3 } \cline { 6 - 7 } \cline { 5 - 7 } & OR & $\mathrm{p}$ Value & & OR & p Value & $95 \% \mathrm{Cl}$ \\
\hline Revascularization & 0.277 & 0.069 & & 0.160 & $0.031 \dagger$ & $0.030-0.845$ \\
\hline Age & 1.009 & 0.762 & & 0.996 & 0.894 & $0.937-1.059$ \\
\hline Male sex & 0.721 & 0.622 & & 0.634 & 0.502 & $0.168-2.394$ \\
\hline Suzuki stage & 0.961 & 0.924 & & 0.549 & 0.268 & $0.190-1.586$ \\
\hline
\end{tabular}

* Statistical analysis was conducted with binominal logistic regression.

$\dagger$ Statistically significant.

of HS was not significantly different in the revascularization group compared with the control group. Because only patients presenting with ischemia were included in this study, the protective effect for HS in hemorrhagic MMD could not be evaluated. However, some evidence suggests that surgical treatments may also have an advantage over nonsurgical ones for the treatment of hemorrhagic MMD. For instance, Miyamoto et al. recently conducted a prospective randomized controlled study that examined the effects of direct revascularization for hemorrhagic MMD. ${ }^{22}$ They reported the annual rebleeding rate as 3.2\% for those who had received surgery and $8.2 \%$ for those who had received nonsurgical treatment. Overall, the role of direct revascularization in preventing recurrent bleeding was supported by statistically significant results. Liu et al. also conducted a retrospective study in patients with hemorrhagic MMD. ${ }^{21}$ The rate of recurrent bleeding was reported to be significantly lower in surgically treated patients, especially in those who had been treated with a direct bypass. Nonetheless, the small number of patients in these studies may limit their reliability.

Despite evidence for a long-term benefit of revascularization surgery, the short- or midterm (5-year) benefit was unclear. This could be mainly due to the incidence of perioperative stroke (Fig. 1). The AS rate curves of the 2 groups crossed at about 60 months, which was likely due to the initial steep slope that was associated with perioperative stroke in the revascularization group. If perioperative strokes were excluded, the annual AS rate in the revascularization group fell to $0.9 \%$ ( $0.4 \%$ for both IS and HS). Perioperative major stroke occurred in $2.7 \%$ of the revascularization group. By comparison, the perioperative stroke rate has been reported to be relatively high (3.4\%$5.6 \%$ ) in previous studies. ${ }^{10,12}$ The prevention of postoperative stroke seems to be the most important factor that makes revascularization for MMD particularly valuable. The importance of controlling perioperative ischemic or hemorrhagic complications has already been well noted in the field of bypass surgery for atherosclerotic disease., ${ }^{7,25,27}$

At the 10-year follow-up point, 1 of 10 patients had experienced a benefit from revascularization surgery compared with hemodynamically stable patients with MMD in terms of preventing further AS. Regarding stroke prevention, NNT has been measured in various treatments. The NNT of oral anticoagulants in nonvalvular atrial fibrillation for the prevention of primary stroke has been reported to be $25 .{ }^{1}$ The NNT of aspirin after acute stroke in the prevention of repeat stroke has been reported to be $146 .{ }^{28}$ Going by these NNT values, we can consider revas-
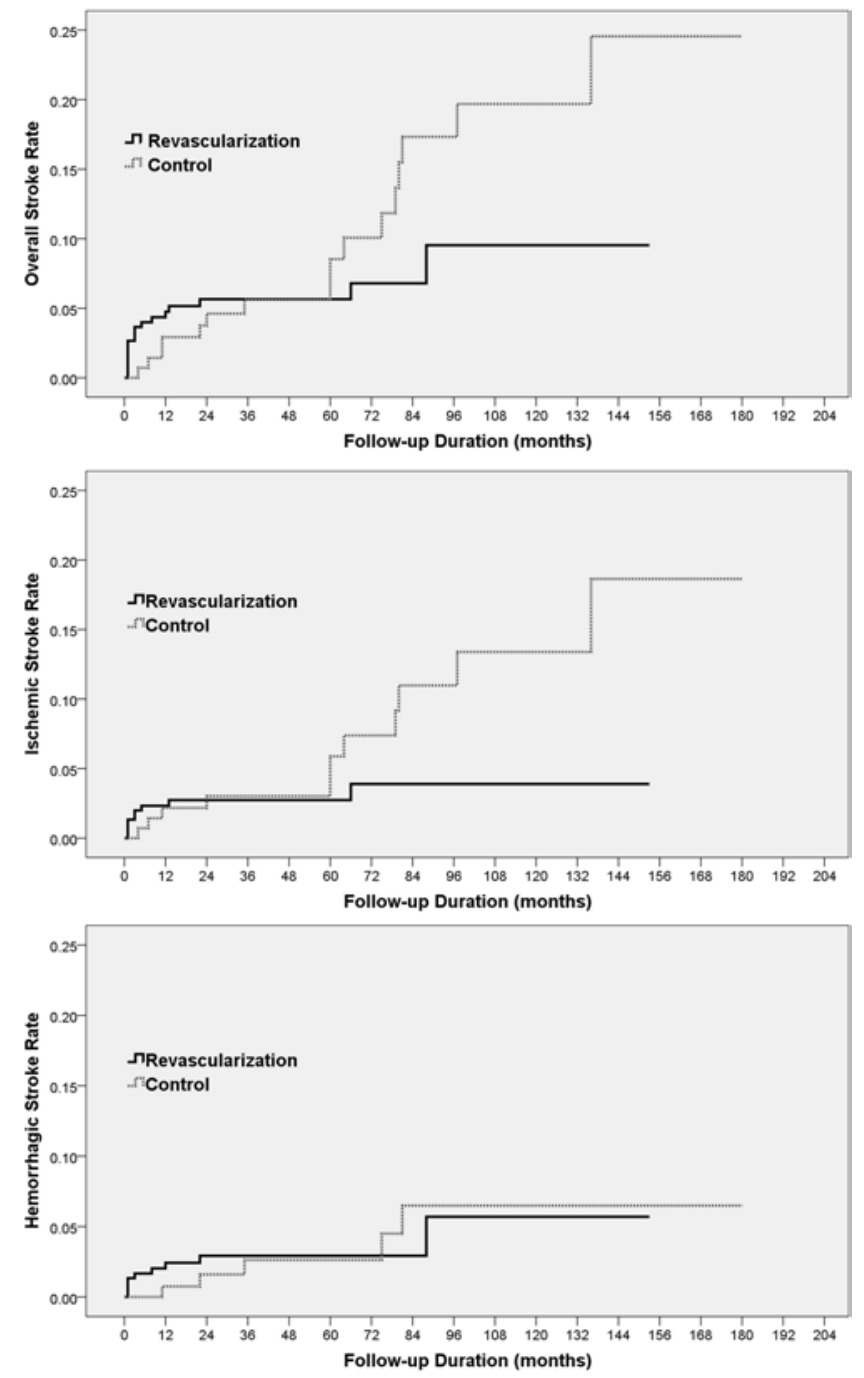

FIG. 1. These graphs show actuarial rates of any stroke (AS; overall stroke rate), ischemic stroke (IS), and hemorrhagic stroke (HS) calculated using the life table method. The steep slope of the curve observed shortly after 0 months shows the perioperative stroke development in the revascularization group.

cularization for hemodynamically unstable MMD to be an effective treatment for preventing future stroke.

The critical limitation of this study is potential allocation bias. Because there had been clear guidelines for selecting patients for surgery, the discrepancy in hemodynamic status was inevitably present between the surgery and control groups. ${ }^{14}$ The hemodynamic status was more compromised in both basal status and reservoir capacity among the surgically treated patients. However, as corrupted hemodynamic status was known to be a risk factor for the recurrence of stroke, this bias could be overcome with proof of the superiority of surgical treatment. Another limitation concerns the retrospective nature of this study. As we have mentioned above, it is difficult to design a prospective controlled study for MMD due to its rare and progressive nature. To produce more reliable evidence for the effects of revascularization treatment of MMD, a more feasible alternative could be to perform a meta-anal- 
ysis using large-scale retrospective studies that include a control group.

\section{Conclusions}

The 10-year actuarial rates of AS and IS were significantly lower in the revascularization group than the control group. Thus, for patients with adult-onset MMD presenting with ischemia, direct or combined revascularization seems to be effective in preventing further stroke.

\section{Acknowledgments}

This study was supported by a grant of the Korea Healthcare Technology R\&D Project, Ministry of Health \& Welfare, Republic of Korea (HI10C2020).

\section{References}

1. Aguilar MI, Hart R: Oral anticoagulants for preventing stroke in patients with non-valvular atrial fibrillation and no previous history of stroke or transient ischemic attacks. Cochrane Database Syst Rev (3):CD001927, 2005

2. Bang JS, Kwon OK, Kim JE, Kang HS, Park H, Cho SY, et al: Quantitative angiographic comparison with the OSIRIS program between the direct and indirect revascularization modalities in adult moyamoya disease. Neurosurgery 70:625-633, 2012

3. Chiu D, Shedden P, Bratina P, Grotta JC: Clinical features of moyamoya disease in the United States. Stroke 29:13471351, 1998

4. Cho WS, Chung YS, Kim JE, Jeon JP, Son YJ, Bang JS, et al: The natural clinical course of hemodynamically stable adult moyamoya disease. J Neurosurg 122:82-89, 2015

5. Cho WS, Kim JE, Kim CH, Ban SP, Kang HS, Son YJ, et al: Long-term outcomes after combined revascularization surgery in adult moyamoya disease. Stroke 45:3025-3031, 2014

6. Duan L, Bao XY, Yang WZ, Shi WC, Li DS, Zhang ZS, et al: Moyamoya disease in China: its clinical features and outcomes. Stroke 43:56-60, 2012

7. The EC/IC Bypass Study Group: Failure of extracranialintracranial arterial bypass to reduce the risk of ischemic stroke. Results of an international randomized trial. N Engl J Med 313:1191-1200, 1985

8. Fujimura M, Tominaga T: Lessons learned from moyamoya disease: outcome of direct/indirect revascularization surgery for 150 affected hemispheres. Neurol Med Chir (Tokyo) 52:327-332, 2012

9. Fukui M: Guidelines for the diagnosis and treatment of spontaneous occlusion of the circle of Willis ('moyamoya' disease). Research Committee on Spontaneous Occlusion of the Circle of Willis (Moyamoya Disease) of the Ministry of Health and Welfare, Japan. Clin Neurol Neurosurg 99 (Suppl 2):S238-S240, 1997

10. Gross BA, Du R: Adult moyamoya after revascularization. Acta Neurochir (Wien) 155:247-254, 2013

11. Gross BA, Du R: The natural history of moyamoya in a North American adult cohort. J Clin Neurosci 20:44-48, 2013

12. Guzman R, Lee M, Achrol A, Bell-Stephens T, Kelly M, Do HM, et al: Clinical outcome after 450 revascularization procedures for moyamoya disease. Clinical article. J Neurosurg 111:927-935, 2009

13. Hallemeier CL, Rich KM, Grubb RL Jr, Chicoine MR, Moran CJ, Cross DT III, et al: Clinical features and outcome in North American adults with moyamoya phenomenon. Stroke 37:1490-1496, 2006

14. Kim JE, Oh CW, Kwon OK, Park SQ, Kim SE, Kim YK: Transient hyperperfusion after superficial temporal artery/ middle cerebral artery bypass surgery as a possible cause of postoperative transient neurological deterioration. Cerebrovasc Dis 25:580-586, 2008
15. Kobayashi E, Saeki N, Oishi H, Hirai S, Yamaura A: Longterm natural history of hemorrhagic moyamoya disease in 42 patients. J Neurosurg 93:976-980, 2000

16. Kraemer M, Berlit P, Diesner F, Khan N: What is the expert's option on antiplatelet therapy in moyamoya disease? Results of a worldwide survey. Eur J Neurol 19:163-167, 2012

17. Kronenburg A, Braun KP, van der Zwan A, Klijn CJ: Recent advances in moyamoya disease: pathophysiology and treatment. Curr Neurol Neurosci Rep 14:423, 2014

18. Kuroda S, Hashimoto N, Yoshimoto T, Iwasaki Y: Radiological findings, clinical course, and outcome in asymptomatic moyamoya disease: results of multicenter survey in Japan. Stroke 38:1430-1435, 2007

19. Kuroda S, Houkin K: Moyamoya disease: current concepts and future perspectives. Lancet Neurol 7:1056-1066, 2008

20. Kuroda S, Houkin K, Ishikawa T, Nakayama N, Iwasaki Y: Novel bypass surgery for moyamoya disease using pericranial flap: its impacts on cerebral hemodynamics and long-term outcome. Neurosurgery 66:1093-1101, 2010

21. Liu X, Zhang D, Shuo W, Zhao Y, Wang R, Zhao J: Long term outcome after conservative and surgical treatment of haemorrhagic moyamoya disease. J Neurol Neurosurg Psychiatry 84:258-265, 2013

22. Miyamoto S, Yoshimoto T, Hashimoto N, Okada Y, Tsuji I, Tominaga T, et al: Effects of extracranial-intracranial bypass for patients with hemorrhagic moyamoya disease: results of the Japan Adult Moyamoya Trial. Stroke 45:1415-1421, 2014

23. Morioka M, Hamada J, Todaka T, Yano S, Kai Y, Ushio Y: High-risk age for rebleeding in patients with hemorrhagic moyamoya disease: long-term follow-up study. Neurosurgery 52:1049-1055, 2003

24. Narisawa A, Fujimura M, Tominaga T: Efficacy of the revascularization surgery for adult-onset moyamoya disease with the progression of cerebrovascular lesions. Clin Neurol Neurosurg 111:123-126, 2009

25. Powers WJ, Clarke WR, Grubb RL Jr, Videen TO, Adams HP Jr, Derdeyn CP: Extracranial-intracranial bypass surgery for stroke prevention in hemodynamic cerebral ischemia: the Carotid Occlusion Surgery Study randomized trial. JAMA 306:1983-1992, 2011 (Erratum in JAMA 306:2672, 2011)

26. Research Committee on the Pathology and Treatment of Spontaneous Occlusion of the Circle of Willis: Guidelines for diagnosis and treatment of moyamoya disease (spontaneous occlusion of the circle of Willis). Neurol Med Chir (Tokyo) 52:245-266, 2012

27. Reynolds MR, Derdeyn CP, Grubb RL Jr, Powers WJ, Zipfel GJ: Extracranial-intracranial bypass for ischemic cerebrovascular disease: what have we learned from the Carotid Occlusion Surgery Study? Neurosurg Focus 36(1):E9, 2014

28. Sandercock PA, Counsell C, Gubitz GJ, Tseng MC: Antiplatelet therapy for acute ischaemic stroke. Cochrane Database Syst Rev (3):CD000029, 2008

\section{Disclosures}

The authors report no conflict of interest concerning the materials or methods used in this study or the findings specified in this paper.

\section{Author Contributions}

Conception and design: all authors. Acquisition of data: T Kim, Cho. Analysis and interpretation of data: T Kim, Cho. Drafting the article: T Kim, Cho. Statistical analysis: T Kim. Study supervision: Bang.

\section{Correspondence}

Jae Seung Bang, Department of Neurosurgery, Seoul National University Bundang Hospital, 82, Gumi-ro 173 Beon-gil, Bundang-gu, Seongnam-si, Gyeonggi-do 463-707, Republic of Korea.email: bang78425@hanmail.net. 